\title{
Bifunctional Silica-Coated Superparamagnetic FePt Nanoparticles for Fluorescence/MR Dual Imaging
}

\author{
Syu-Ming Lai, ${ }^{1}$ Tsiao-Yu Tsai, ${ }^{1}$ Chia-Yen Hsu, ${ }^{1}$ Jai-Lin Tsai, ${ }^{2}$ \\ Ming-Yuan Liao, ${ }^{1}$ and Ping-Shan Lai ${ }^{1}$ \\ ${ }^{1}$ Department of Chemistry, National Chung Hsing University, 250 Kuo Kuang Road, Taichung 402, Taiwan \\ ${ }^{2}$ Department of Materials Science and Engineering, National Chung Hsing University, 250 Kuo Kuang Road, Taichung 402, Taiwan
}

Correspondence should be addressed to Ping-Shan Lai, pslai@email.nchu.edu.tw

Received 14 January 2012; Revised 26 March 2012; Accepted 26 March 2012

Academic Editor: Krasimir Vasilev

Copyright (C) 2012 Syu-Ming Lai et al. This is an open access article distributed under the Creative Commons Attribution License, which permits unrestricted use, distribution, and reproduction in any medium, provided the original work is properly cited.

Recently, superparamagnetic chemically disordered face-centered cubic (fcc) FePt nanoparticles have been demonstrated as superior negative contrast agents for magnetic resonance imaging (MRI). However, their low intracellular labeling efficiency has limited the potential usage and the nanotoxicity of the particles requires attention. We have developed fluorescein isothiocyanate-incorporated silica-coated FePt (FePt@SiO 2 -FITC) nanoparticles that exhibited not only a significant $T_{1}$ and $T_{2}$ MR contrast abilities but also a fluorescent property without significant cytotoxicities. These results suggest that silica-coated superparamagnetic FePt nanoparticles are potential nanodevices for the combination of fluorescence and MRI contrast used for cancer diagnosis.

\section{Introduction}

Superparamagnetic iron oxide (SPIO) nanoparticles have demonstrated their practicability as MRI $T_{2}$-shortening agents for noninvasive cell labeling, drug delivery or tumor detections in clinical practice $[1,2]$. However, low intracellular labeling efficiency and nanotoxicity of SPIO has limited their potential usage $[3,4]$. Recently, bifunctional contrast agents for both optical and MR imaging have been developed to function as good imaging probes in vitro and in vivo [5$8]$ and the nanotoxicity of SPIO is suppressed by particular surface modification [9]. Thus, proper modifications of SPIO have been considered as a promising strategy to improve the aforementioned problems.

Long-range ordered $L 1_{0}$ FePt films have been studied extensively and thought to be a promising candidate for ultrahigh-density magnetic recording media due to its high magnetocrystalline anisotropy $\left(K_{\mathrm{u}}\right)$. The magnetization thermal instability or superparamagnetic effect is delayed due to their very small critical grain size of around $(3-5 \mathrm{~nm})$. However, when FePt nanoparticles are disordered with facecentered cubic (fcc) structure, they exhibit superparamagnetic property under critical size. Superparamagnetic iron-platinum (FePt) nanoparticles, which show high saturation magnetization $\left(M_{S}\right)$ compared to SPIO, are expected to be a high performance nanomagnet for magnetic medicine $[10,11]$. In particular, the potential of FePt nanoparticles as an MRI contrast agent has been demonstrated [12]. Thus, the bifunctional contrast agent using superparamagnetic FePt nanoparticles seems to be a promising dual-modality biomedical detections material. In our strategy, silica was selected for surface coating of FePt nanoparticles to enhance the biocompatibility and easier modifications for bioconjugation or cell targeting [13]. Moreover, fluorescent dyes can be easily incorporated into a silica shell that provides a powerful tool for intracellular tracking [14]. Hence, the bifunctional fluorescein-isothiocyanate-(FITC-) incorporated silica-coated superparamagnetic FePt $\left(\mathrm{FePt} @ \mathrm{SiO}_{2}-\right.$ FITC) nanoparticles were synthesized and their potential applications for dual fluorescent/magnetic imaging in this study were evaluated.

\section{Experimental Procedure}

2.1. Materials. For materials preparation, iron pentacarbonyl $\left(\mathrm{Fe}(\mathrm{CO})_{5}, 99.99 \%\right)$, platinum(II) acetylacetonate 


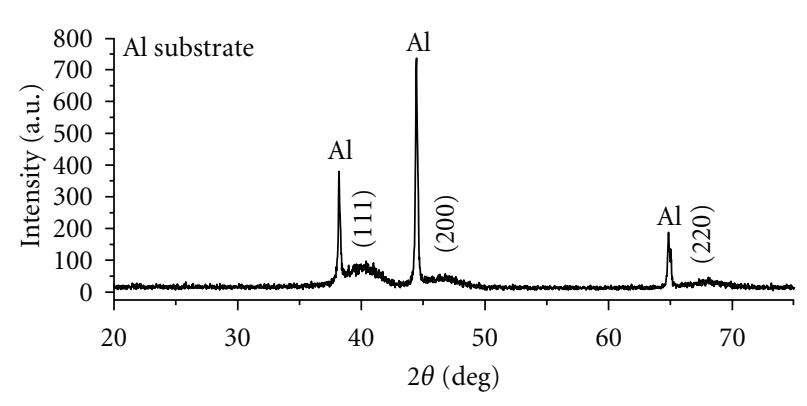

(a) $\mathrm{FePt}$

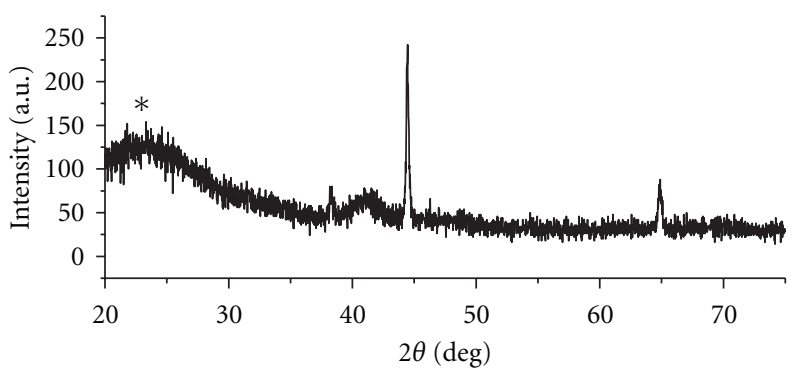

(b) $\mathrm{FePt@SiO} 2$-FITC

FIgURE 1: XRD patterns of FePt and FePt@SiO ${ }_{2}$-FITC nanoparticles.

(Pt $(\text { acac })_{2}$, 97\%, 1,2-hexadecanediol, florescence isocyanate (FITC), 3-Aminopropyltrimethoxysilane (APTMS, 97\%), Triton X100, hexanol, and benzyl ether were purchased from Sigma-Aldrich (St. Louis, MO, USA). Oleylamine (80-90\%) was obtained from Acros (New Jersey, USA). Oleic acid and tetraethyl silicate (TEOS, 99.9\%) were obtained from Showa (Tokyo, Japan). The ethanol (99.5\%) and hexane were purchased from Echo in Taiwan. The solvents were all dehydrated prior to use.

For cell culture studies, sodium phosphate dibasic $\left(\mathrm{Na}_{2} \mathrm{HPO}_{4}\right)$ and sodium chloride $(\mathrm{NaCl})$ were obtained from Tedia $(\mathrm{OH}, \mathrm{USA})$. Potassium chloride $(\mathrm{KCl})$, potassium dihydrogenphosphate $\left(\mathrm{KH}_{2} \mathrm{PO}_{4}\right)$, and sodium bicarbonate $\left(\mathrm{NaHCO}_{3}\right)$ were obtained from Showa (Tokyo, Japan). Modified Eagle's Medium (MEM), fetal bovine serum (FBS), and $0.25 \%$ trypsin-EDTA were purchased from Gibco (GibcoBRL, USA). The penicillin-streptomycin-neomycin solution and $10 \%$ formalin were purchased from Sigma-Aldrich (St. Louis, MO, USA). Double distilled water was used in all of the aqueous solutions.

2.2. Synthesis of FePt Nanoparticles. The synthesis of fcc FePt nanoparticles involving simultaneous chemical reduction of $\mathrm{Pt}(\mathrm{acac})_{2}$ and $\mathrm{Fe}(\mathrm{CO})_{5}$ by 1,2-hexadecanediol at high temperature was described as previous report [15]. Briefly, $\mathrm{Pt}(\mathrm{acac})_{2} \quad(0.48 \mathrm{mmol})$ was dissolved in $10 \mathrm{~mL}$ of benzyl ether and the solution was heated to $100^{\circ} \mathrm{C}$ under nitrogen atmosphere. The surfactants of oleylamine $(0.5 \mathrm{mmol})$ and oleic acid $(0.5 \mathrm{mmol})$ were added into the reaction and subsequently the $\mathrm{Fe}(\mathrm{CO})_{5}(1 \mathrm{mmol})$ was injected. The mixture solution was then heated to $297^{\circ} \mathrm{C}$ under refluxing for 30 minutes. Finally, the reaction system was cooled down to room temperature and the FePt nanoparticles were purified using ethanol washing and then collected by centrifugation.

2.3. Synthesis of Silica-Coated FePt Nanoparticles. The bifunctional magnetic silica-coated FePt $\left(\mathrm{FePt} @ \mathrm{SiO}_{2}\right)$ nanoparticles were synthesized by a water-in-oil microemulsion method as previous reports $[13,16]$. First, the $N-1-(3-$ triethoxysilylpropyl)- $N$-fluoresceyl thiourea (FITC-APTMS) was prepared by stirring $5 \mathrm{mg}$ FITC in $5 \mathrm{~mL}$ ethanolic APTMS solution ( $10 \mathrm{v} / \mathrm{v} \%)$ for 24 hours. The monodisperse hydrophobic FePt nanoparticles were also redissolved in cyclohexane at room temperature and Triton X-100, hexanol and distilled water were added into the solution with stirring to generate a microemulsion. Then the TEOS, APTMS, and FITC-labeled APTMS were added into the system to form the functional silica shell structure. The sol-gel growth of silica was limited in the water domain in this w/o microemulsion. All procedures were carried out in subdued light. To remove free surfactants and unreacted chemicals, the as-synthesized silica-coated FePt nanoparticles solution was centrifuged and washed with ethanol and deionized water. All procedures were repeated twice and the final silicacoated FePt nanoparticles were stored in deionized water for following experiments.

These FePt@SiO 2 -FITC nanoparticles were evaluated by the X-ray diffraction (XRD), transmission electron microscope (TEM), vibration sample magnetometer (VSM), fluorescence spectroscopy, $T_{2}$ enhancing relaxivity, and determinate the iron content of $\mathrm{FePt@SiO} \mathrm{S}_{2}$-FITC nanoparticles by inductively coupled plasma atomic emission spectroscopy (ICP-AES).

2.4. Characterizations of FePt Nanoparticles and FePt@SiO ${ }_{2}-$ FITC Nanoparticles. The FePt nanoparticles were further characterized by XRD (Bruker MXP-III) with a $2.0 \mathrm{~kW} \mathrm{Cu}$ tube and a Sol-X energy dispersive detector. The XRD sample was mounted with Vaseline on a glass substrate, and the data were collected from $20^{\circ}-80^{\circ} 2 \theta$ ( step size $=0.6^{\circ}$ and time per step $=1 \mathrm{~s})$ at room temperature.

The size and morphologies were observed under the TEM (JEM 1200, JEOL Ltd., Japan). Samples for TEM analysis were prepared as dilute dispersions in hexane/water with a small amount of surfactants. The size distributions were evaluated by dynamic light scattering (DLS, ZS-90, Malvern).

The magnetic properties and performance were characterized by VSM. Magnetization curves as a function of applied field were measured with fields up to $1.2 \mathrm{kOe}$ at room temperatures. The relaxation times $\left(T_{1}\right.$ and $\left.T_{2}\right)$ were measured by NMR (300 MHz, Varian) at room temperature. The iron concentrations of samples were determined by ICPAES (ICAP 9000, Jarrell-Ash, USA). The MRI images were all taken by the clinical 3T MR scanner (Signa Excite 3 T, GE Healthcare, USA).

The cytotoxicity of FePt and FePt@SiO 2 -FITC nanoparticles was evaluated by MTT assay using human cervical 


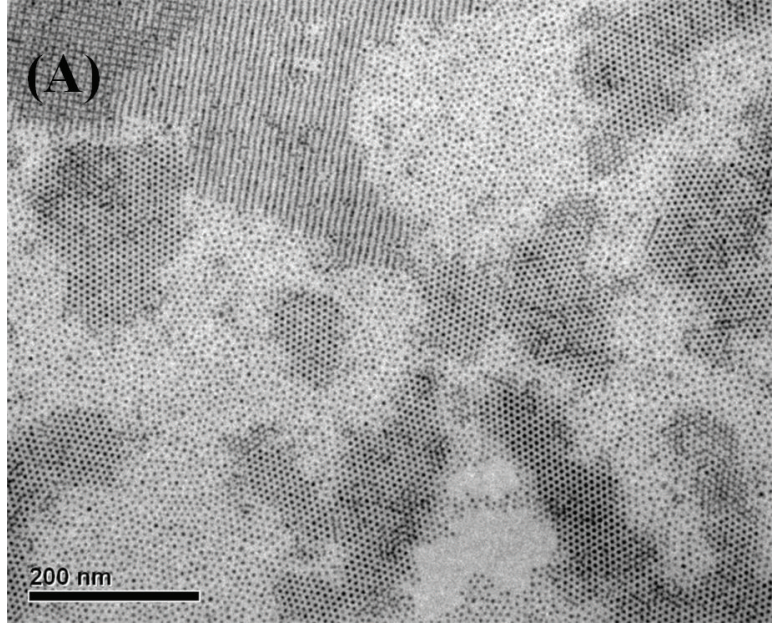

(a)

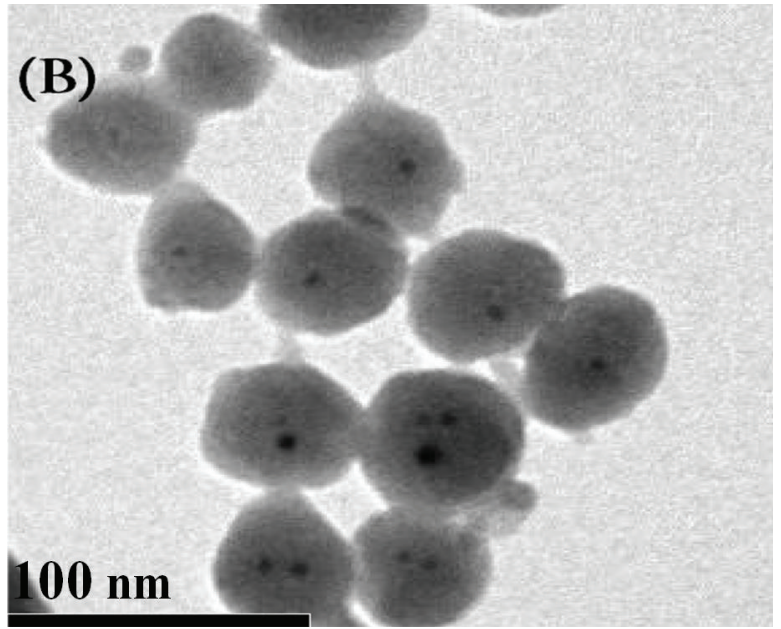

(b)

FIgUre 2: The TEM images of (a) FePt and (b) FePt@SiO 2 -FITC nanoparticles.

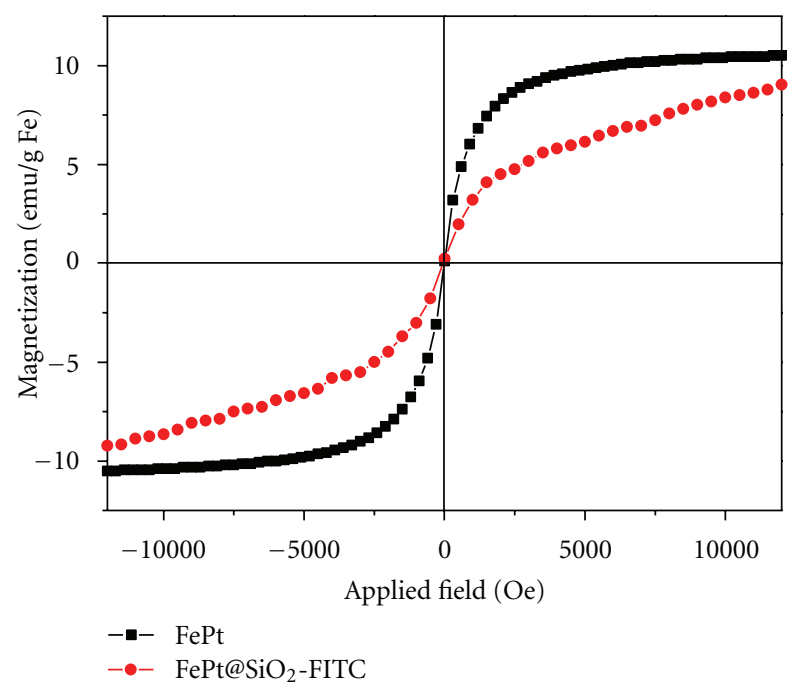

FIgURe 3: Room-temperature magnetization curves of FePt and FePt@SiO 2 -FITC nanoparticles.

epithelioid carcinoma (HeLa) cells. The intracellular localization of FePt@SiO 2 -FITC nanoparticles in HeLa cells was examined using a confocal microscope after costaining with LysoTracker Red (Invitrogen) as our previous report [17].

\section{Results and Discussions}

FePt nanoparticles were synthesized by heating the $\mathrm{Pt}(\mathrm{acac})_{2}$ and $\mathrm{Fe}(\mathrm{CO})_{5}$ precursors with the surfactants at $297^{\circ} \mathrm{C}$ for 30 minutes to form the black suspension. The as-synthesized FePt nanoparticles were washed and centrifuged for the following characterization and evaluations. Figure 1 showed the XRD patterns of oleic acid/oleylamine-capped FePt nanocrystals with or without the functionalized $\mathrm{SiO}_{2}$ coating. It is known that the FePt nanoparticles were
TABLE 1: Longitudinal and transverse relaxivities and relaxivity ratios of FePt and FePt@SiO 2 -FITC nanoparticles.

\begin{tabular}{lccc}
\hline Material & $r_{1}\left(\mathrm{~s}^{-1} \mathrm{mM}^{-1}\right)$ & $r_{2}\left(\mathrm{~s}^{-1} \mathrm{mM}^{-1}\right)$ & $r_{2} / r_{1}$ \\
\hline FePt & 5.7 & 396.1 & 69.5 \\
FePt@SiO $_{2}$-FITC & 6.8 & 102.3 & 15.0 \\
\hline
\end{tabular}

generated rapidly as the temperature rises from 250 to $297^{\circ} \mathrm{C}$. Saita and Maenosono had also reported the effects of heating rate on the synthesized FePt nanoparticles [18]. Thus, we set the reaction temperature at $297^{\circ} \mathrm{C}$ for $30 \mathrm{~min}$ in this study to avoid formation of $\gamma-\mathrm{Fe}_{2} \mathrm{O}_{3}$ (or $\mathrm{Fe}_{3} \mathrm{O}_{4}$ ). As shown in Figure 1(a), the (111), (200), and (220) peaks of typical chemically disordered fcc-phase FePt crystallite without being annealed were evidently observed and there was no (311) peak observed around $2 \theta=35^{\circ}$ [18-20]. These FePt nanocrystals were coated with $\mathrm{SiO}_{2}$ using waterin-oil microemulsions method and $\mathrm{NH}_{4} \mathrm{OH}$ was used to catalyze the decomposition of TEOS to silica over the course of 48 hrs. XRD patterns of $\mathrm{FePt} @ \mathrm{SiO}_{2}$-FITC nanoparticles confirmed the existence of fcc-phase FePt after $\mathrm{SiO}_{2}$ coating (Figure 1(b)). Due to the amorphous $\mathrm{SiO}_{2}$ coating, the broaden peak was found in the low diffraction angle (indicated by ${ }^{*}$ ) [21]. It is noticed that the (111) FePt fundamental peak was shift slightly to the high angle. To prepare the FePt@SiO 2 and $\mathrm{FePt}_{2} @ \mathrm{SiO}_{2}$-FITC nanoparticles, the multiple-surface modifications were carried out under air condition, and thus the surface oxidation of FePt might be occurred. In the XRD study, the shifted peak to high angle (from $\sim 40^{\circ}$ to $\sim 41.7^{\circ}$ ) may be due to the coupling of FePt crystallite with the oxidized product on the $\mathrm{FePt}$ nanoparticle surface, in which the peak at about 41.7 is near to the reflection peak of $\mathrm{FeO}(200)$ at $\sim 42^{\circ}$ according to the assignment of JPCDS No. 86-2316 [22, 23]. Further investigations of FePt nanoparticles for structure changes are being undertaken at our lab. 


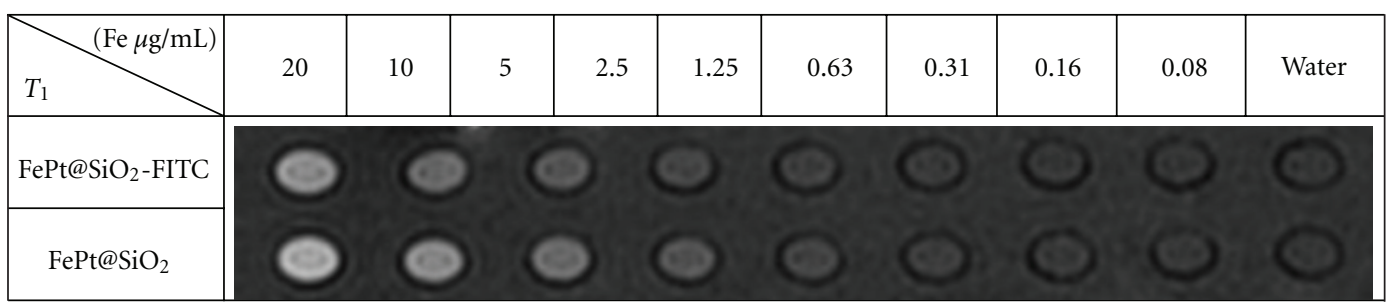

(a)

\begin{tabular}{|c|c|c|c|c|c|c|c|c|c|c|}
\hline$(\mathrm{Fe} \mu \mathrm{g} / \mathrm{mL})$ & 20 & 10 & 5 & 2.5 & 1.25 & 0.63 & 0.31 & 0.16 & 0.08 & Water \\
\hline FePt@SiO & & &
\end{tabular}

(b)

FIgURE 4: The MRI images of FePt@SiO ${ }_{2}$ and FePt@SiO 2 -FITC nanoparticles evaluated by clinical 3T MR scanner.

Figure 2 shows the TEM images of FePt and FePt@SiO ${ }_{2}-$ FITC nanoparticles and the sizes were analyzed by SigmaScan Pro software. The size of FePt nanoparticles was $4.59 \pm 0.80 \mathrm{~nm}$ with sphere/cubic shape (Figure 2(a)). The $\mathrm{FePt} @ \mathrm{SiO}_{2}$-FITC nanoparticles revealed $48.98 \pm 6.41 \mathrm{~nm}$ thick silica structure that was coated around several FePt nanoparticles from TEM observation (Figure 2(b)). The polydispersity of FePt@SiO 2 -FITC nanoparticles observed by DLS was 0.182 .

The room-temperature magnetic hysteresis curves of FePt and FePt@SiO 2 -FITC nanoparticles were all normalized to emu per gram of Fe content as shown in Figure 3. Clearly, the fcc-phase FePt nanoparticles sustain their superparamagnetic property in the silica composites at room temperature [20]. However, the lower and unsaturated magnetization of FePt@SiO 2 -FITC nanoparticles observed under the applied field of $1.2 \mathrm{~T}$ may be due to the surface loose density of FePt component in the silanized particles or the surface dead layer around $\mathrm{SiO}_{2}$ [24-26]. The significant difference in $M_{S}$ among nanoparticles capped with varied ligands and the dead layer thickness have been estimated as previous report [27]. Thus, the electron donation from ligands to the Fe d band may reduce the magnetization. The possible $\mathrm{FeO}$ formation may also reduce $\mathrm{Ms}$ of nanoparticles.

The $T_{1}$ and $T_{2}$ values obtained from FePt or FePt@SiO FITC nanoparticles dispersed in pure water are summarized in (Table S1 and Figure S1 in Supplementary Material available online at doi:10.1155/2012/631584) and the inverse relaxation times were almost linearly proportional to the concentration of nanoparticle similar to previous report [12]. Consequently, the $r_{1}$ (longitudinal relaxivities) and $r_{2}$ (transverse relaxivities) values of dispersed in pure water were determined to be 5.8 and 396.1 for FePt or 6.8 and $102.3 \mathrm{~s}^{-1} \mathrm{mM}^{-1}$ for FePt@SiO $\mathrm{S}_{2}$-FITC nanoparticles, respectively (Table 1). For a $T_{2}$ contrast agent, a higher $r_{2} / r_{1}$ ratio has a better contrast efficacy [28] and thus FePt@SiO ${ }_{2}$ FITC nanoparticles with $r_{2} / r_{1}=15$ can be potentially used for MR contrast. The MRI images of FePt@SiO ${ }_{2}$ and $\mathrm{FePt} @ \mathrm{SiO}_{2}$-FITC nanoparticles were evaluated by clinical $3 \mathrm{~T}$ MR scanner and the results were shown in Figure 4. Unexpectedly, FePt@SiO 2 and FePt@SiO 2 -FITC nanoparticles revealed bright $T_{1}$-weighted imaging, whereas $T_{2}$ weighted imaging of both nanoparticles became dark as the Fe concentration increased. FePt nanoparticle has been demonstrated as potential $T_{2}$-weighted contrast agent for MR imaging due to its magnetization $[29,30]$. For the $T_{1}$ contrast enhancement, it is possible that the generation of $\mathrm{FeO}$ surface coating on the FePt nanoparticle may provide additional assistance for the spin-lattice relaxation process.

The fluorescence spectrum of FePt@SiO 2 -FITC nanoparticles was shown in Figure S1. Clearly, the property of FITC was observed in FePt@SiO 2 -FITC nanoparticles which exhibited strong fluorescence at $535 \mathrm{~nm}$ with the excitation wavelength at $488 \mathrm{~nm}$. Thus, FePt@SiO ${ }_{2}$-FITC nanoparticles could be observed directly inside the cells by the florescence of the FITC. Figure 5 showed the intracellular localization of FePt@SiO 2 -FITC nanoparticles in HeLa cells. After 12 hours incubation, the colocalization of fluorescence (Figure 5(c)) of FePt@SiO 2 -FITC nanoparticles (Figure 5(a)) and LysoTracker (Figure 5(b)) was observed in HeLa cells. It is speculated that FePt@SiO $\mathrm{S}_{2}$-FITC nanoparticles were internalized into cells and entrapped presumably in the endosomes/lysosomes, as suggested by confocal microscopic observation. This result indicated that the FePt@SiO ${ }_{2}$-FITC nanoparticles might be taken up by cells via endocytosis. The dose-dependent cytotoxicity of FePt and $\mathrm{FePt}_{\mathrm{SiO}}$ FITC in HeLa cells was demonstrated in Figure 6. No significant cytotoxicity was observed after $500 \mu \mathrm{M}$ FePt@SiO $2_{-}^{-}$ FITC nanoparticles incubation for $24 \mathrm{~h}$ or $72 \mathrm{~h}$, whereas FePt nanoparticles induced cell toxicity at $500 \mu \mathrm{M}$ for $72 \mathrm{~h}$ incubation (62\% cell survival). Thus, the cytotoxicity of FePt nanoparticles was potentially suppressed with silica coating.

The introduction of APTMS and FITC-labeled APTMS in synthetic procedure of silica-coated nanoparticle not only 


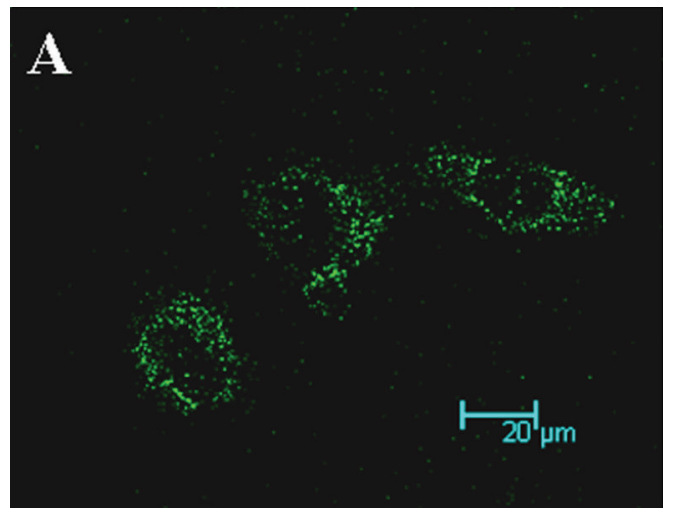

(a)

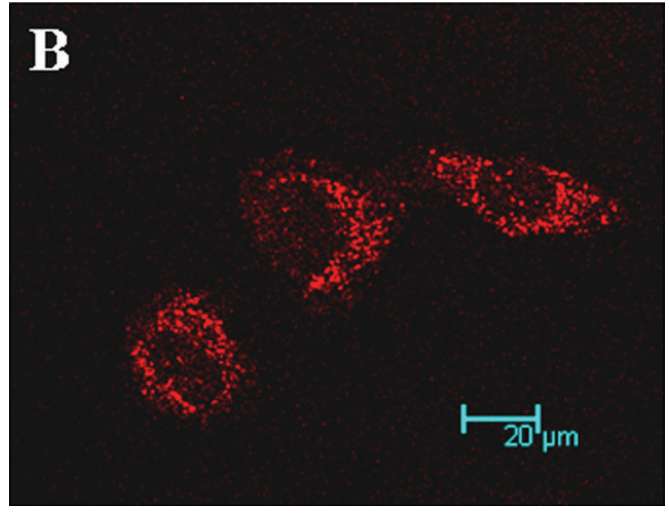

(b)

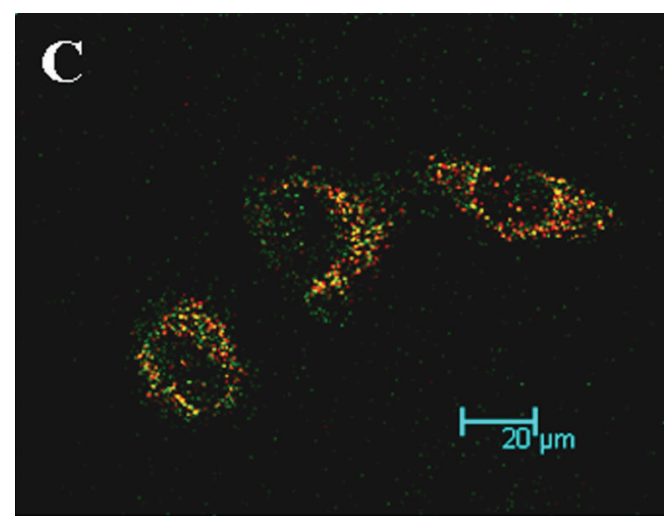

(c)

Figure 5: Comparative intracellular localization of FePt@SiO 2 -FITC nanoparticles with LysoTracker Red observed by confocal laser scanning microscopy.

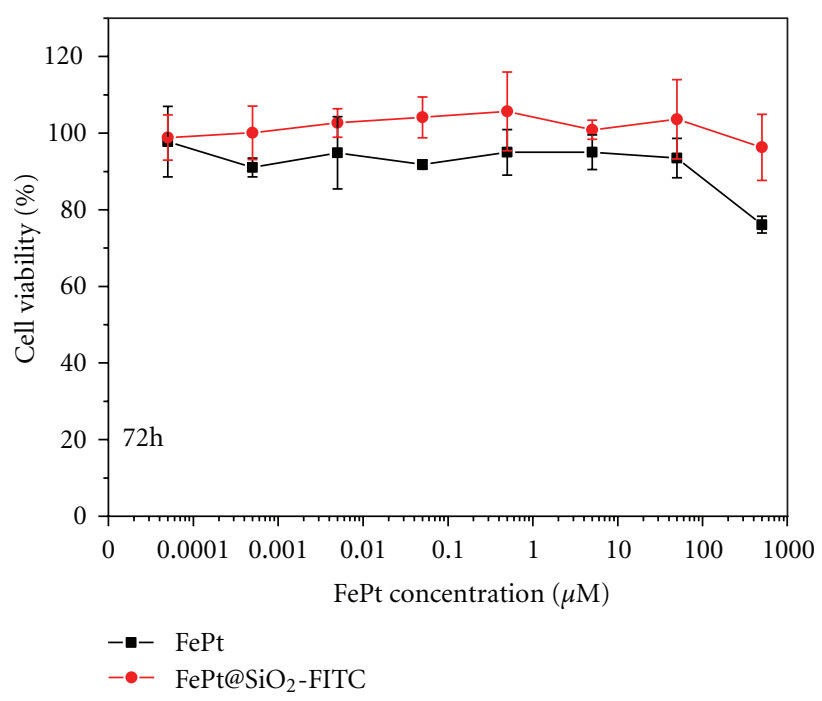

(a)

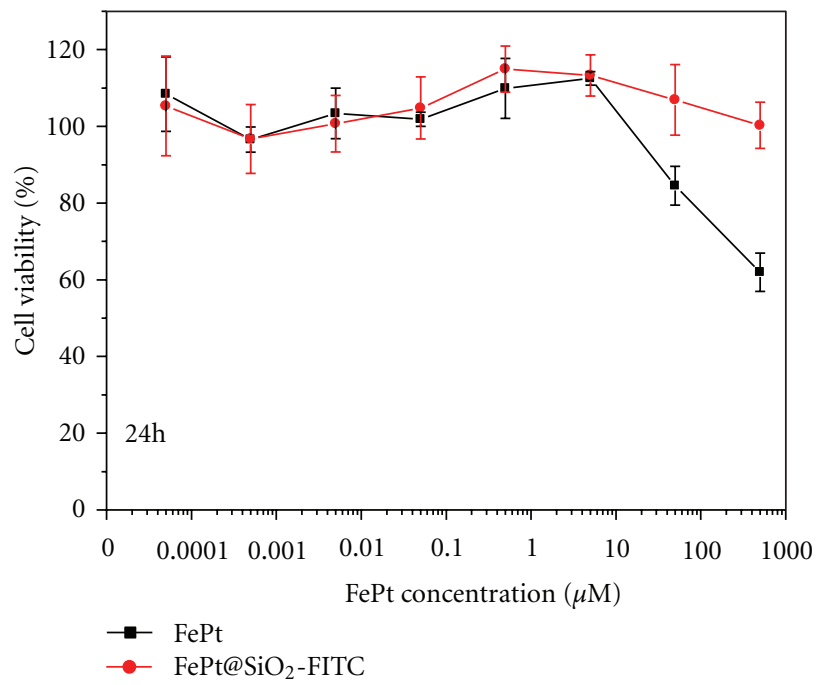

(b)

FIgURe 6: Cytotoxicity of FePt and FePt@SiO 2 -FITC nanoparticles. Cells were incubated for $24 \mathrm{~h}$ or $72 \mathrm{~h}$ at $37^{\circ} \mathrm{C}(n=4)$. 
provide the fluorescent property but produce the positive surface charge of FePt@SiO ${ }_{2}$-FITC nanoparticles (Figure S2) that facilitate attachment and internalization of the particles [31]. Moreover, the amino groups of APTMS can be further conjugated using hydrophilic polyethylene glycol that results in steric hindrance to the phagocyte, system, and prolongation of blood circulation time [32]. Developing multiple imaging modalities in one nanoparticle is a promising field for biomedical applications. Chou et al. reported the dual modal biomedical imaging by using the $\mathrm{FePt}$ nanoparticles as simultaneous CT and MRI contrast agent [29]. Malvindi et al. also reported the dual mode imaging of the FePt-iron oxide and silica nanoparticles combination as the MRI and ultrasonography contrast agent [30]. These reports showed the advantages of these highly potential dualmode imaging contrast agents for biomedical applications. Further investigations of FePt@SiO ${ }_{2}$-FITC nanoparticles for simultaneous fluorescence/MR imaging in vivo are ongoing at our lab.

\section{Conclusion}

The longitudinal and transverse proton relaxation times obtained with silica-coated superparamagnetic FePt nanoparticles was measured. Unexpectedly, $\mathrm{FePt} @ \mathrm{SiO}_{2}$ and FePt@SiO ${ }_{2}$-FITC nanoparticles revealed bright $T_{1}$-weighted imaging, whereas $T_{2}$-weighted imaging of both nanoparticles became dark as the Fe concentration increased. Moreover, the surface coating using silica successfully suppressed the nanotoxicity of FePt. These results suggest that silica-coated superparamagnetic FePt nanoparticles are potential nanodevices for the combination of simultaneous fluorescence and MRI contrast for cancer diagnosis.

\section{Acknowledgment}

This research was supported by grants from Ministry of Education of the Republic of China under the ATU plan and National Science Council of the Republic of China (NSC 1002218-E-002-002 and 100-2628-E-005-008-MY2).

\section{References}

[1] J. V. Frangioni, "New technologies for human cancer imaging," Journal of Clinical Oncology, vol. 26, no. 24, pp. 4012-4021, 2008.

[2] N. Nasongkla, E. Bey, J. Ren et al., "Multifunctional polymeric micelles as cancer-targeted, MRI-ultrasensitive drug delivery systems," Nano Letters, vol. 6, no. 11, pp. 2427-2430, 2006.

[3] N. Lewinski, V. Colvin, and R. Drezek, "Cytotoxicity of nanopartides," Small, vol. 4, no. 1, pp. 26-49, 2008.

[4] T. R. Pisanic, J. D. Blackwell, V. I. Shubayev, R. R. Fiñones, and S. Jin, "Nanotoxicity of iron oxide nanoparticle internalization in growing neurons," Biomaterials, vol. 28, no. 16, pp. 25722581, 2007.

[5] C. Tu, Y. Yang, and M. Gao, "Preparations of bifunctional polymeric beads simultaneously incorporated with fluorescent quantum dots and magnetic nanocrystals," Nanotechnology, vol. 19, no. 10, Article ID 105601, 2008.
[6] H. M. Liu, S. H. Wu, C. W. Lu et al., "Mesoporous silica nanoparticles improve magnetic labeling efficiency in human stem cells," Small, vol. 4, no. 5, pp. 619-626, 2008.

[7] W. J. M. Mulder, R. Koole, R. J. Brandwijk et al., "Quantum dots with a paramagnetic coating as a bimodal molecular imaging probe," Nano Letters, vol. 6, no. 1, pp. 1-6, 2006.

[8] C. Zhang and X. Xie, "Controllable assembly of hydrophobic superparamagnetic iron oxide nanoparticle with mPEG-PLA copolymer and its effect on MR transverse relaxation rate," Journal of Nanomaterials, vol. 2011, Article ID 152524, 7 pages, 2011.

[9] A. K. Gupta and M. Gupta, "Cytotoxicity suppression and cellular uptake enhancement of surface modified magnetic nanoparticles," Biomaterials, vol. 26, no. 13, pp. 1565-1573, 2005.

[10] S. Sun, C. B. Murray, D. Weller, L. Folks, and A. Moser, "Monodisperse FePt nanoparticles and ferromagnetic FePt nanocrystal superlattices," Science, vol. 287, no. 5460, pp. 19891992, 2000.

[11] S. Maenosono and S. Saita, "Theoretical assessment of FePt nanoparticles as heating elements for magnetic hyperthermia," IEEE Transactions on Magnetics, vol. 42, no. 6, pp. 16381642, 2006.

[12] S. Maenosono, T. Suzuki, and S. Saita, "Superparamagnetic FePt nanoparticles as excellent MRI contrast agents," Journal of Magnetism and Magnetic Materials, vol. 320, no. 9, pp. L79L83, 2008.

[13] J. R. Skuza, R. A. Lukaszew, E. M. Dufresne et al., "Real time structural modification of epitaxial FePt thin films under x-ray rapid thermal annealing using undulator radiation," Applied Physics Letters, vol. 90, no. 25, Article ID 251901, 2007.

[14] L. Wang and W. Tan, "Multicolor FRET silica nanoparticles by single wavelength excitation," Nano Letters, vol. 6, no. 1, pp. 84-88, 2006.

[15] S. Sun, E. E. Fullerton, D. Weller, and C. B. Murray, "Compositionally controlled FePt nanoparticle materials," IEEE Transactions on Magnetics, vol. 37, no. 4, pp. 1239-1243, 2001.

[16] T. Tago, T. Hatsuta, K. Miyajima, M. Kishida, S. Tashiro, and K. Wakabayashi, "Novel synthesis of silica-coated ferrite nanoparticles prepared using water-in-oil microemulsion," Journal of the American Ceramic Society, vol. 85, no. 9, pp. 2188-2194, 2002.

[17] M. J. Shieh, C. L. Peng, P. J. Lou et al., "Non-toxic phototriggered gene transfection by PAMAM-porphyrin conjugates," Journal of Controlled Release, vol. 129, no. 3, pp. 200-206, 2008.

[18] S. Saita and S. Maenosono, "Formation mechanism of FePt nanoparticles synthesized via pyrolysis of iron(III) ethoxide and platinum(II) acetylacetonate," Chemistry of Materials, vol. 17, no. 26, pp. 6624-6634, 2005.

[19] H. L. Nguyen, L. E. M. Howard, G. W. Stinton et al., "Synthesis of size-controlled fcc and fct FePt nanoparticles," Chemistry of Materials, vol. 18, no. 26, pp. 6414-6424, 2006.

[20] L. Colak and G. C. Hadjipanayis, "Phase transformation in silica-coated FePt nanoparticles," IEEE Transactions on Magnetics, vol. 45, no. 10, pp. 4081-4084, 2009.

[21] G. Gupta, M. N. Patel, D. Ferrer et al., "Stable ordered FePt mesoporous silica catalysts with high loadings," Chemistry of Materials, vol. 20, no. 15, pp. 5005-5015, 2008.

[22] Y. Hou, Z. Xu, and S. Sun, "Controlled synthesis and chemical conversions of $\mathrm{FeO}$ nanoparticles," Angewandte Chemie International Edition, vol. 46, no. 33, pp. 6329-6332, 2007.

[23] T. T. Trinh, D. Mott, N. T. K. Thanh, and S. Maenosono, "Onepot synthesis and characterization of well defined core-shell 
structure of FePt@CdSe nanoparticles," RSC Advances, vol. 1, pp. 100-108, 2011.

[24] E. Kockrick, F. Schmidt, K. Gedrich et al., "Mesoporous ferromagnetic MPt@Silica/Carbon ( $=$ Fe, Co, Ni) composites As advanced bifunctional catalysts," Chemistry of Materials, vol. 22, no. 5, pp. 1624-1632, 2010.

[25] P. J. Chen, S. H. Hu, C. S. Hsiao, Y. Y. Chen, D. M. Liu, and S. Y. Chen, "Multifunctional magnetically removable nanogated lids of $\mathrm{Fe}_{3} \mathrm{O}_{4}$-capped mesoporous silica nanoparticles for intracellular controlled release and MR imaging," Journal of Materials Chemistry, vol. 21, no. 8, pp. 2535-2543, 2011.

[26] P. E. Le Renard, R. Lortz, C. Senatore et al., "Magnetic and in vitro heating properties of implants formed in situ from injectable formulations and containing superparamagnetic iron oxide nanoparticles (SPIONs) embedded in silica microparticles for magnetically induced local hyperthermia," Journal of Magnetism and Magnetic Materials, vol. 323, no. 8, pp. 1054-1063, 2011.

[27] Y. Tanaka, S. Saita, and S. Maenosono, "Influence of surface ligands on saturation magnetization of FePt nanoparticles," Applied Physics Letters, vol. 92, no. 9, Article ID 093117, 2008.

[28] J. Qin, S. Laurent, Y. S. Jo et al., "A high-performance magnetic resonance imaging $T_{2}$ contrast agent," Advanced Materials, vol. 19, no. 14, pp. 1874-1878, 2007.

[29] S. W. Chou, Y. H. Shau, P. C. Wu, Y. S. Yang, D. B. Shieh, and C. C. Chen, "In vitro and in vivo studies of fept nanoparticles for dual modal CT/MRI molecular imaging," Journal of the American Chemical Society, vol. 132, no. 38, pp. 13270-13278, 2010.

[30] M. A. Malvindi, A. Greco, F. Conversano et al., "Magnetic/silica nanocomposites as dual-mode contrast agents for combined magnetic resonance imaging and ultrasonography," Advanced Functional Materials, vol. 21, no. 13, pp. 2548-2555, 2011.

[31] P. S. Lai, C. L. Pai, C. L. Peng, M. J. Shieh, K. Berg, and P. J. Lou, "Enhanced cytotoxicity of saporin by polyamidoamine dendrimer conjugation and photochemical internalization," Journal of Biomedical Materials Research A, vol. 87, no. 1, pp. 147-155, 2008.

[32] H. Otsuka, Y. Nagasaki, and K. Kataoka, "PEGylated nanoparticles for biological and pharmaceutical applications," Advanced Drug Delivery Reviews, vol. 55, no. 3, pp. 403-419, 2003. 

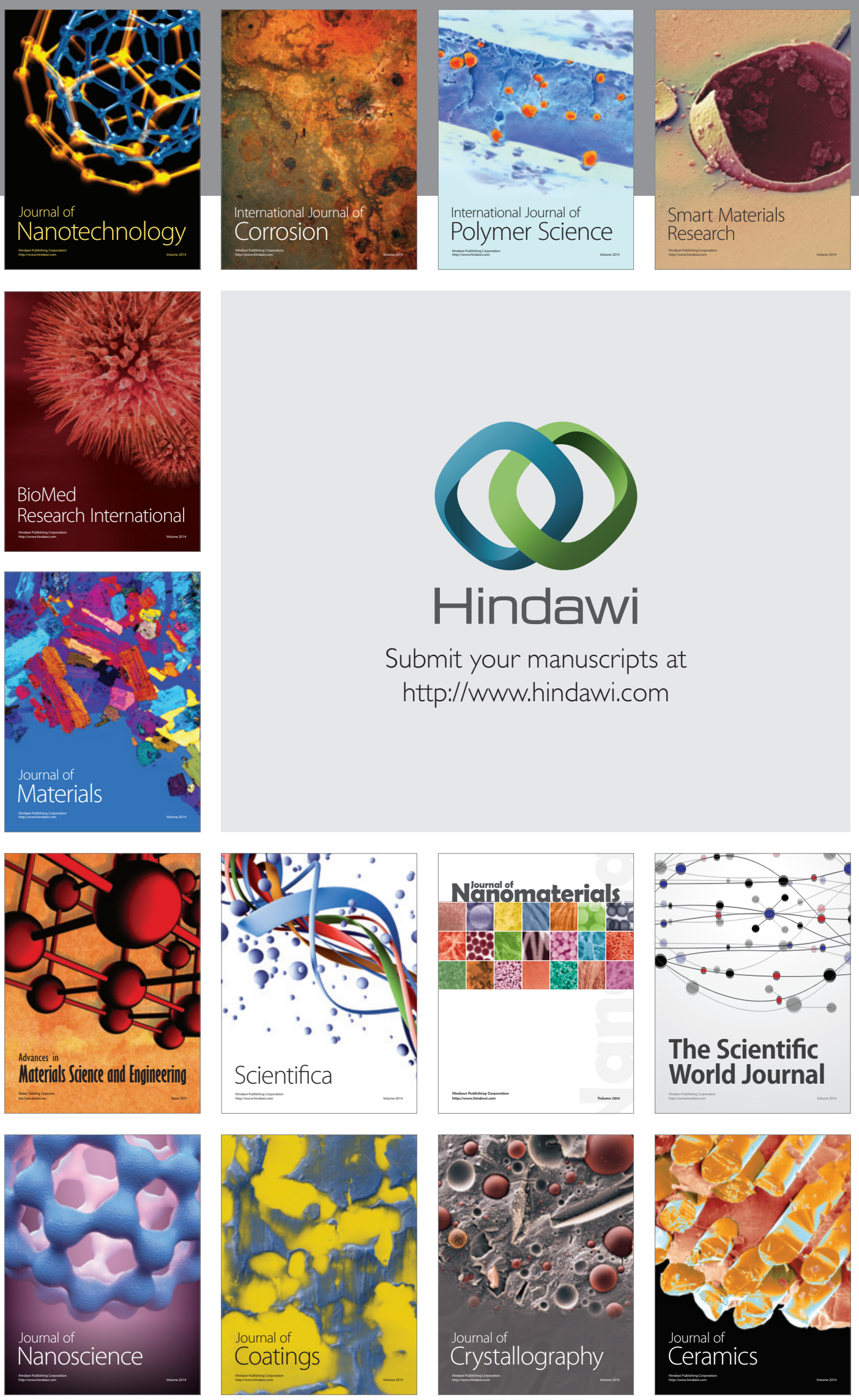

The Scientific World Journal

Submit your manuscripts at

http://www.hindawi.com

\section{World Journal}

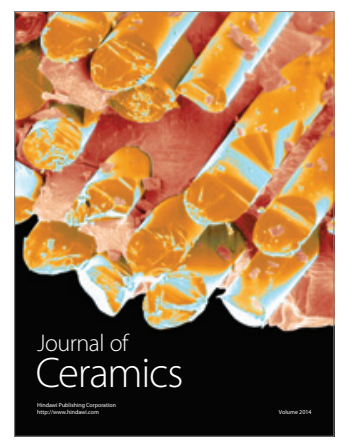

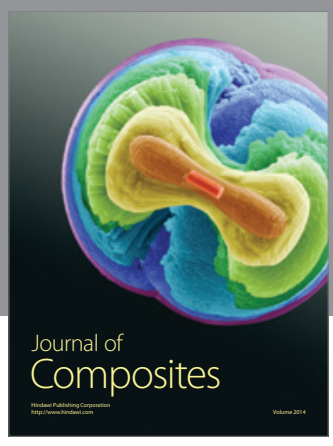
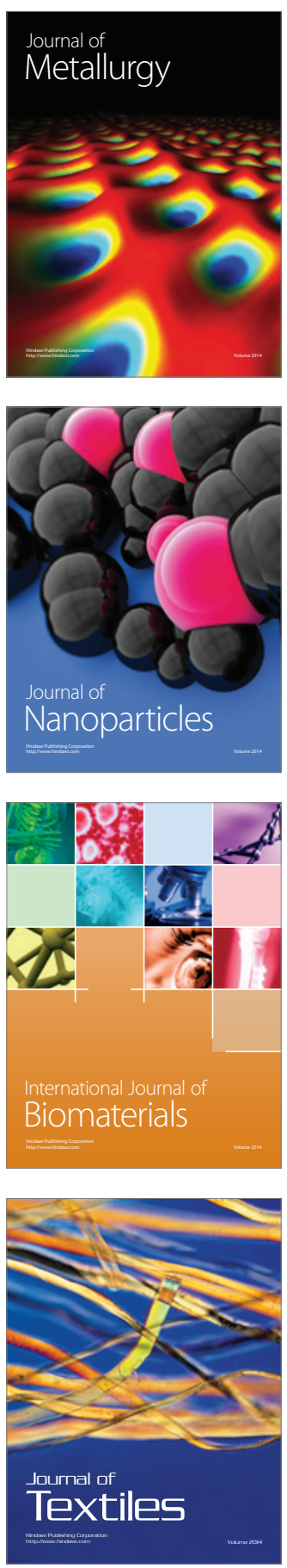